\title{
Distribution, prevalence, and intensity of the swim bladder parasite Anguillicola crassus in New England and eastern Canada
}

\author{
Amy E. Aieta, Kenneth Oliveira* \\ Department of Biology, University of Massachusetts Dartmouth, Dartmouth, Massachusetts 02747, USA
}

\begin{abstract}
In the summer of 2005, yellow phase American eels Anguilla rostrata were examined for the swim bladder parasite Anguillicola crassus from 26 locations in New England, USA, ranging from the Pawcatuck River, Rhode Island, to the East Machias River, Maine. An additional 12 sites were sampled within Canada during the summers of 2006 and 2007: 7 sites in southern Nova Scotia and 5 sites within the St. Lawrence River system. In 2007, eels were also obtained from New Brunswick, northern Nova Scotia, Prince Edward Island, and Newfoundland through the commercial eel fishery. All locations in Rhode Island $(n=3)$ and Massachusetts $(n=10)$ and 7 in Maine $(n=13)$ had infected eels, with parasite prevalence ranging from 7 to $76 \%$. No eels sampled from southern Nova Scotia or the St. Lawrence River system were infected with the parasite. New Brunswick and northern Nova Scotia had infected eels ranging from 3 to $30 \%$ parasite prevalence, with Cape Breton, Nova Scotia, being the furthest north the parasite has been reported in American eels. There was no significant relationship between parasite prevalence and latitude. The present study supports the hypothesis that the parasite is capable of expanding its range further into the Maritimes and could potentially reach the St. Lawrence River system.
\end{abstract}

KEY WORDS: Anguilla rostrata • Anguillicola crassus · Parasite · Swimbladder • Range • New England · Canada

\section{INTRODUCTION}

The American eel Anguilla rostrata is the only species of Anguilla that lives in North America, occupying freshwater, estuarine, and marine habitats (Tesch 2003). Historically, it has been an extremely successful species, in part, because of its large native range, great diversity of habitats, and dietary generalist behavior (Helfman et al. 1987). Recent evidence suggests that the $A$. rostrata population is declining at the northern extent of its range, particularly in the Lake OntarioSt. Lawrence River area (Castonguay et al. 1994) and in the USA (Richkus \& Whalen 1999). Several factors have been suggested as contributing to eel decline: chemical contamination, habitat modifications, commercial fishing, and oceanic changes (Castonguay et al. 1994, Haro et al. 2000, Friedland et al. 2007). The recent introduction of the swim bladder parasite $A n$ - guillicola crassus into North America is another factor that must now be considered (Haro et al. 2000).

Anguillicola crassus is a nematode that establishes itself in the swim bladder of an anguillid eel via ingestion of an infected intermediate freshwater ostracod (Pietrock \& Meinelt 2002, Moravec 2004), calanoid (Pietrock \& Meinelt 2002), cyclopoid copepod (De Charleroy et al. 1990), or one of many paratenic fish species (Johnson et al. 1995). In the European eel Anguilla anguilla, the parasite has been shown to cause an array of serious problems that include internal bleeding (Mellergaard \& Dalsgaard 1989), inflammatory reactions (Molnár 1994) and dilation of blood vessels, formation of granuloma, and thickening of the entire swim bladder wall (Würtz \& Taraschewski 2000). Swim bladders have often been found to be irreversibly damaged due to the lumen becoming filled with dead A. crassus, digested blood, and debris, as 
well as the swim bladder wall becoming full of encapsulated adults and juveniles (Kirk 2003). Studies examining the effects of the parasite on American eels are limited. Ooi et al. (1996) found infected American eels raised in a Taiwanese fish farm had hemorrhaged and ruptured swim bladders, anemia, and peritonitis due to internal parasite migration. Similarly, Sokolowski \& Dove (2006) found pathological conditions in infected eels to include blood-filled swim bladders and deleterious alterations of the submucosa, mucularis, serosa, and lamina propria of the swim bladder wall.

When Japanese eels were imported from Asia to Europe for evaluation in aquaculture in the 1980s (Johnson et al. 1995), the imported eels led to the establishment of Anguillicola crassus in the European eel (Gollock et al. 2004). More recently, the parasite has been identified in the American eel. Although the first reports were from a Texas aquaculture facility in 1994 (Johnson et al. 1995), subsequent investigation showed the source of these eels to be elvers transferred from South Carolina (Fries et al. 1996), indicating an east coast origin for the parasite. In 1997, fishers found A. crassus in American eels in a tributary in the midChesapeake Bay and, subsequently, in tributaries of the Chesapeake Bay and Hudson River (Barse \& Secor 1999), as well as within the Hudson River estuary (Morrison \& Secor 2003).

During the summer of 2003, Anguillicola crassus was discovered in yellow eels from the Paskamansett River, Dartmouth, Massachussetts (K. Oliveira unpubl. data). The parasite had never been recorded this far north in the American eel range. Considering the potential effects of this introduction on American eels and the parasite's history of rapid range expansion, the present study was conducted to explore the current distribution of the parasite in New England, the Maritime Provinces, and the St. Lawrence River.

\section{MATERIALS AND METHODS}

During the summer of 2005, yellow eels Anguilla rostrata were collected from 26 sites within New England, 3 of the sites were in Rhode Island, 10 in Massachusetts, and 13 in Maine (Fig. 1, see Table 1). Sampling further south was not deemed necessary as it had been determined that the parasite was already established within the Connecticut River (S. Gephard, CT Department of Environmental Protection, pers. comm.). During the summer of 2006, yellow eels were collected from 5 rivers in Nova Scotia and 5 locations within the St. Lawrence River system, 3 being tributaries and 2 being dams in the main stem of the river (Fig. 1, see Table 1). Eels were collected from all riverine sites by electrofishing from June to September. Sites were sim- ilar in general characteristics; all were coastal and above the head of tide (freshwater), depth $<50 \mathrm{~cm}$, low to moderate flow, but varied between predominantly sandy to predominantly rocky substrate. Due to difficulty accessing freshwater areas within the Kennebec River, Maine, 25 estuarine yellow phase eels were obtained from eel pots set in the estuary. Eels collected from the Beauharnois Dam in Quebec and the MosesSaunders Dam in Ontario were captured while ascending the eel ladders at those facilities. Latitude and longitude were recorded at each site using a handheld GPS unit. Only yellow phase eels, the primary feeding stage, were collected during electrofishing and dam collections.

In the summer of 2007, 4 rivers in Nova Scotia were resampled (Beaver, Salmon, Metaghan, and Coques) and 2 additional rivers (Sissiboo and West) were considered to expand the sampling range (Fig. 1, see Table 1). In addition, commercially collected eels from the Maritime Provinces of New Brunswick, northern Nova Scotia, Prince Edward Island (PEI), and Newfoundland were also sampled at the South Shore Trading processing plant (Port Elgin, New Brunswick) (Fig. 1, see Table 2). Whole eels from PEI and Newfoundland were not available, so our examination of these locations was limited to whole freshly removed swim bladders.

After collection, eels were killed by immersion in clove oil (Eugenol). Eel weight $(g)$ and total length $(\mathrm{mm})$ were recorded. The swim bladder was dissected 0 to $6 \mathrm{~d}$ after collection and opened for analysis of infection. Eels not dissected within $24 \mathrm{~h}$ of collection were slit from anus to head and placed in 70 to $80 \%$ isopropyl alcohol for temporary preservation until examination. The swim bladders from PEI and Newfoundland eels were preserved in $70 \%$ isopropyl and then examined using the same procedures as swim bladders from whole eels. Eels were assessed for prevalence, i.e. the numbers of infected eels divided by the total number of eels examined, and mean intensity, i.e. the average number of nematodes found among the infected eels (Bush et al. 1997). Only nematodes found in the lumen were examined in the present study. These intact and partial lumen nematodes were counted and placed in vials containing 70 to $85 \%$ ethanol. Parasite identifications were made using a dissecting microscope and the descriptions of Kuwahara et al. (1974), Moravec \& Taraschewski (1988), and Moravec (2004). Voucher specimens were deposited at the US National Parasite Collection (USNPC No. 101411.00).

Data from the eels from the fishery samples were known to have several limitations: (1) the location of capture was limited to the river or general region and no GPS data were available, (2) the eels were all of commercial size $(>300 \mathrm{~mm})$ and not representative of 


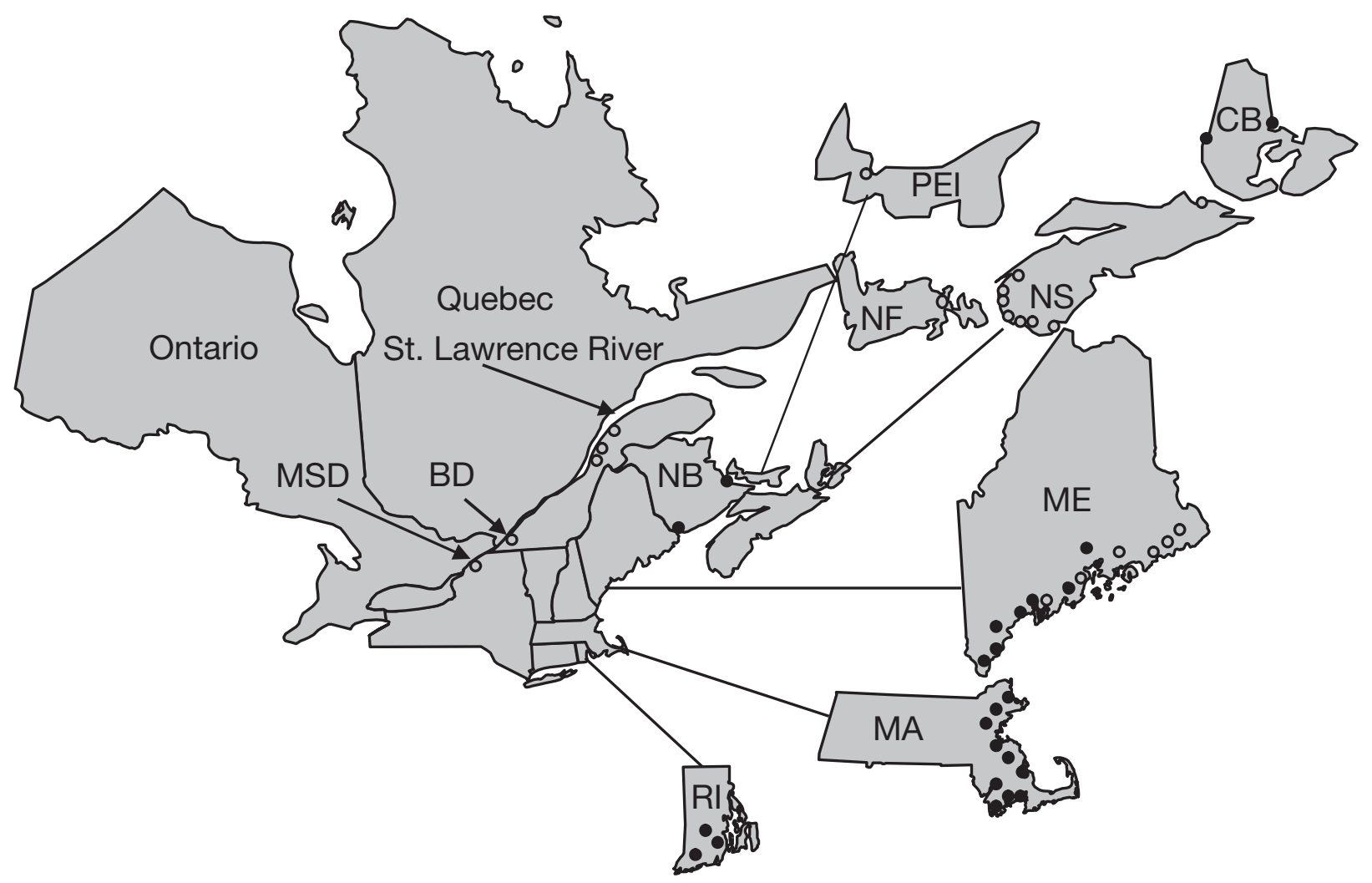

Fig. 1. Collection locations of American eels Anguilla rostrata in the United States and Canada. BD: Beauharnois Dam in Quebec; CB: Cape Breton Island; MA: Massachusetts; ME: Maine; MSD: Moses Saunders Dam in Ontario; NB: New Brunswick; NF: Newfoundland; NS: Nova Scotia; PEI: Prince Edward Island; RI: Rhode Island; O: sites without the parasite Anguillicola crassus;

- sites with the parasite

the overall size distribution of the sampling location, and (3) only swim bladders were available for eels from PEI and Newfoundland.

Data were analyzed with SPSS 13.0 for Windows. The Pearson correlation model was used to examine the relationship between latitude and prevalence and to explore relationships between prevalence and intensity. Significance was accepted for all tests at the $\mathrm{p} \leq 0.05$ level.

\section{RESULTS}

The parasite Anguillicola crassus had a consistent distribution throughout most of the New England sampling locations; it was found at all 3 sites in Rhode Island and at all 10 sites in Massachusetts, with the maximum prevalence being $76 \%$ in the Neponset River, Massachussetts. The prevalence of infection in Maine ranged from $0 \%$ to a high of $65 \%$ in eels Anguilla rostrata collected from the Kennebunk River (Table 1). The northernmost site in the USA with infected eels was the Sedgeunkedunk Stream, a tributary of the Penobscot River, Maine. The parasite exhib- ited a more disjunctive distribution in Canadian locations, with no parasites being found at locations within the St. Lawrence River or in southern Nova Scotia. A single parasite was found within an eel from the St. Johns River and Silver Lake, New Brunswick, but several parasitized eels were present in northern Nova Scotia (Table 2).

Due to data limitations, the distribution analysis was limited to the New England sites. Correlation analysis of the relationship between latitude and the prevalence of infection was limited to the range of the parasite (Pawcatuck River, Rhode Island, to Penobscot River, Maine), with the Penobscot latitude adjusted to the mouth of the river $\left(44^{\circ} 22.150^{\prime} \mathrm{N}\right)$. The Nova Scotia locations were also omitted to remove any complicating longitudinal component from the analysis. This analysis revealed no significant correlation between latitude and the prevalence of infection (Pearson correlation $=-0.32$, 2-tailed significance $p=0.147$ ). There was a strong positive correlation between the prevalence of infection and mean intensity (Pearson correlation $=0.81$, 2-tailed significance $\mathrm{p}<0.001$ ), confirming that sites of higher prevalence had higher mean parasite intensity. 
Yellow eels, collected from all locations, ranged in length from 56 to $699 \mathrm{~mm}(\mathrm{n}=1324$ eels; mean $\pm \mathrm{SD}=$ $248.8 \pm 101.4 \mathrm{~mm}$ ). Infected eels ranged in length from 70 to $594 \mathrm{~mm}(\mathrm{n}=315$ eels; mean $\pm \mathrm{SD}=242.9 \pm$ $88.5 \mathrm{~mm}$ ), with parasite intensity ranging from 1 to 18 parasites eel ${ }^{-1}$ (mean $\pm \mathrm{SD}=3.2 \pm 2.8$ ). Non-parasitized eels from locations that had parasitized eels ranged in size from 56 to $699 \mathrm{~mm}(\mathrm{n}=403$ eels; mean $\pm \mathrm{SD}=$ $203.9 \pm 93.8 \mathrm{~mm})$. The significant difference in mean eel size among rivers prevented comparisons of prevalence and intensity from being made among locations.

\section{DISCUSSION}

Data from all collections indicate that the parasite Anguillicola crassus has at least spread to Cape Breton Island, Nova Scotia, on the east coast of North America.

Table 1. Anguillicola crassus, Anguilla rostrata. Summary of the prevalence and mean intensity of A. crassus in eels A. rostrata for all locations sampled in the USA and Canada. Values for intensity, length and weight are mean \pm SD and range (in parentheses)

\begin{tabular}{|c|c|c|c|c|c|c|c|c|c|}
\hline \multirow[t]{2}{*}{ Location } & \multirow{2}{*}{$\begin{array}{l}\text { Lat., } \\
\text { Long. }\end{array}$} & \multicolumn{2}{|c|}{ No. of eels (n) } & \multirow{2}{*}{$\begin{array}{c}\text { Prevalence } \\
(\%)\end{array}$} & \multirow[t]{2}{*}{ Intensity } & \multicolumn{2}{|c|}{ Eel length (mm) } & \multicolumn{2}{|c|}{ Eel weight (g) } \\
\hline & & Collected & Infected & & & Non-infected & Infected & Non-infected & Infected \\
\hline \multicolumn{10}{|l|}{ Rhode Island } \\
\hline $\begin{array}{l}\text { Pawcatuck } \\
\text { River }\end{array}$ & $\begin{array}{l}41^{\circ} 26.27^{\prime} \mathrm{N} \\
71^{\circ} 43.32^{\prime} \mathrm{W}\end{array}$ & 29 & 20 & 69 & $\begin{array}{c}2.6 \pm 2.4 \\
(1-11)\end{array}$ & $\begin{array}{c}125.3 \pm 8.3 \\
(112-137)\end{array}$ & $\begin{array}{c}183.1 \pm 111.5 \\
(102-530)\end{array}$ & $\begin{array}{c}2.6 \pm 0.8 \\
(1-4)\end{array}$ & $\begin{array}{c}27.7 \pm 73.6 \\
(2-324)\end{array}$ \\
\hline $\begin{array}{l}\text { Gilbert Stuart } \\
\text { Brook }\end{array}$ & $\begin{array}{l}41^{\circ} 31.18^{\prime} \mathrm{N}, \\
71^{\circ} 26.67^{\prime} \mathrm{W}\end{array}$ & 71 & 25 & 35 & $\begin{array}{l}2.2 \pm 1.6 \\
(1-7)\end{array}$ & $\begin{array}{c}202.5 \pm 73.0 \\
(56-375)\end{array}$ & $\begin{array}{c}245.8 \pm 59.1 \\
(125-327)\end{array}$ & $\begin{array}{c}8.0 \pm 19.1 \\
(1-90)\end{array}$ & $\begin{array}{c}26.8 \pm 17.2 \\
(2-60)\end{array}$ \\
\hline $\begin{array}{l}\text { Annaquatucket } \\
\text { River }\end{array}$ & $\begin{array}{l}41^{\circ} 33.03^{\prime} \mathrm{N}, \\
71^{\circ} 26.45^{\prime} \mathrm{W}\end{array}$ & 29 & 8 & 28 & $\begin{array}{l}3.5 \pm 1.9 \\
(1-6)\end{array}$ & $\begin{array}{c}232.9 \pm 79.9 \\
(77-322)\end{array}$ & $\begin{array}{c}232.8 \pm 84.5 \\
(142-376)\end{array}$ & $\begin{array}{l}29.5 \pm 22.4 \\
\quad(1-75)\end{array}$ & $\begin{array}{c}28.3 \pm 26.5 \\
(3-75)\end{array}$ \\
\hline \multicolumn{10}{|l|}{ Massachusetts } \\
\hline Slocums River & $\begin{array}{l}41^{\circ} 34.29^{\prime} \mathrm{N} \\
71^{\circ} 00.27^{\prime} \mathrm{W}\end{array}$ & 69 & 44 & 64 & $\begin{array}{c}2.9 \pm 1.7 \\
(1-8)\end{array}$ & $\begin{array}{c}244.7 \pm 59.6 \\
(138-335)\end{array}$ & $\begin{array}{c}234.2 \pm 45.5 \\
(160-310)\end{array}$ & $\begin{array}{c}17.2 \pm 22.9 \\
(4-74)\end{array}$ & $\begin{array}{c}13.3 \pm 21.3 \\
(5-64)\end{array}$ \\
\hline Westport River & $\begin{array}{l}41^{\circ} 38.03^{\prime} \mathrm{N}, \\
71^{\circ} 03.82^{\prime} \mathrm{W}\end{array}$ & 33 & 11 & 33 & $\begin{array}{c}2.8 \pm 1.6 \\
(1-5)\end{array}$ & $\begin{array}{c}185.8 \pm 40.6 \\
(114-250)\end{array}$ & $\begin{array}{c}177.1 \pm 52.5 \\
(70-252)\end{array}$ & $\begin{array}{c}9.8 \pm 6.5 \\
(2-23)\end{array}$ & $\begin{array}{c}9.8 \pm 8.4 \\
(1-29)\end{array}$ \\
\hline Acushnet River & $\begin{array}{l}41^{\circ} 41.01^{\prime} \mathrm{N}, \\
70^{\circ} 55.17^{\prime} \mathrm{W}\end{array}$ & 45 & 26 & 58 & $\begin{array}{l}3.6 \pm 3.9 \\
(1-18)\end{array}$ & $\begin{array}{c}276.1 \pm 125.5 \\
(92-699)\end{array}$ & $\begin{array}{c}276.2 \pm 57.1 \\
(140-362)\end{array}$ & $\begin{array}{c}61.2 \pm 127.6 \\
(1-580)\end{array}$ & $\begin{array}{c}41.9 \pm 21.6 \\
(4-79)\end{array}$ \\
\hline $\begin{array}{l}\text { Weweantic } \\
\text { River }\end{array}$ & $\begin{array}{l}41^{\circ} 46.79^{\prime} \mathrm{N}, \\
70^{\circ} 45.79^{\prime} \mathrm{W}\end{array}$ & 19 & 4 & 21 & $\begin{array}{c}3.3 \pm 2.6 \\
(1-7)\end{array}$ & $\begin{array}{c}235.3 \pm 55.8 \\
(155-322)\end{array}$ & $\begin{array}{c}288.0 \pm 64.5 \\
(156-299)\end{array}$ & $\begin{array}{c}21.6 \pm 17.3 \\
(4-57)\end{array}$ & $\begin{array}{c}21.1 \pm 19.3 \\
(5-45)\end{array}$ \\
\hline Eel River & $\begin{array}{l}41^{\circ} 55.06^{\prime} \mathrm{N}, \\
70^{\circ} 37.58^{\prime} \mathrm{W}\end{array}$ & 43 & 23 & 53 & $\begin{array}{c}3.1 \pm 2.0 \\
(1-7)\end{array}$ & $\begin{array}{c}276.6 \pm 83.3 \\
(127-492)\end{array}$ & $\begin{array}{c}295.0 \pm 131.6 \\
(126-594)\end{array}$ & $\begin{array}{c}48.0 \pm 48.1 \\
(2-206)\end{array}$ & $\begin{array}{c}76.6 \pm 98.1 \\
(3-345)\end{array}$ \\
\hline South River & $\begin{array}{l}42^{\circ} 05.86^{\prime} \mathrm{N}, \\
70^{\circ} 43.07^{\prime} \mathrm{W}\end{array}$ & 44 & 7 & 16 & $\begin{array}{c}3.0 \pm 1.2 \\
(2-5)\end{array}$ & $\begin{array}{c}193.2 \pm 77.9 \\
(66-343)\end{array}$ & $\begin{array}{c}253.3 \pm 63.9 \\
(116-320)\end{array}$ & $\begin{array}{l}8.4 \pm 20.2 \\
(1-85)\end{array}$ & $\begin{array}{c}31.1 \pm 18.5 \\
(2-65)\end{array}$ \\
\hline Neponset River & $\begin{array}{l}42^{\circ} 15.73^{\prime} \mathrm{N}, \\
71^{\circ} 02.83^{\prime} \mathrm{W}\end{array}$ & 37 & 28 & 76 & $\begin{array}{c}5.3 \pm 4.2 \\
(1-14)\end{array}$ & $\begin{array}{c}223.0 \pm 66.0 \\
(116-297)\end{array}$ & $\begin{array}{c}108.1 \pm 57.1 \\
(113-329)\end{array}$ & $\begin{array}{l}27.1 \pm 20.4 \\
(2-59)\end{array}$ & $\begin{array}{c}21.1 \pm 18.7 \\
(2-78)\end{array}$ \\
\hline Danvers River & $\begin{array}{l}42^{\circ} 34.20^{\prime} \mathrm{N} \\
70^{\circ} 55.68^{\prime} \mathrm{W}\end{array}$ & 21 & 13 & 62 & $\begin{array}{c}3.5 \pm 2.7 \\
(1-9)\end{array}$ & $\begin{array}{c}183.5 \pm 58.1 \\
(112-282)\end{array}$ & $\begin{array}{c}235.4 \pm 101.7 \\
(85-392)\end{array}$ & $\begin{array}{c}11.6 \pm 12.5 \\
(1-37)\end{array}$ & $\begin{array}{c}33.7 \pm 40.0 \\
(1-118)\end{array}$ \\
\hline $\begin{array}{l}\text { Annisquam } \\
\text { River }\end{array}$ & $\begin{array}{l}42^{\circ} 36.59^{\prime} \mathrm{N} \\
70^{\circ} 42.54^{\prime} \mathrm{W}\end{array}$ & 27 & 9 & 33 & $\begin{array}{c}2.0 \pm 1.3 \\
(1-5)\end{array}$ & $\begin{array}{c}108.7 \pm 72.4 \\
(61-389)\end{array}$ & $\begin{array}{c}198.6 \pm 102.7 \\
(101-381)\end{array}$ & $\begin{array}{c}6.3 \pm 24.1 \\
(1-103)\end{array}$ & $\begin{array}{c}24.0 \pm 37.1 \\
(1-99)\end{array}$ \\
\hline Parker River & $\begin{array}{l}42^{\circ} 44.97^{\prime} \mathrm{N} \\
70^{\circ} 55.71^{\prime} \mathrm{W}\end{array}$ & 56 & 4 & 7 & $\begin{array}{c}1.8 \pm 1.5 \\
(1-4)\end{array}$ & $\begin{array}{c}133.8 \pm 89.6 \\
(71-376)\end{array}$ & $\begin{array}{c}134.0 \pm 100.3 \\
(77-284)\end{array}$ & $\begin{array}{c}9.4 \pm 18.7 \\
(1-87)\end{array}$ & $\begin{array}{c}8.6 \pm 16.3 \\
(1-33)\end{array}$ \\
\hline \multicolumn{10}{|l|}{ Maine } \\
\hline York River & $\begin{array}{l}43^{\circ} 10.39^{\prime} \mathrm{N} \\
70^{\circ} 46.28^{\prime} \mathrm{W}\end{array}$ & 30 & 16 & 53 & $\begin{array}{c}3.4 \pm 2.6 \\
(1-10)\end{array}$ & $\begin{array}{c}210.1 \pm 53.7 \\
(100-296)\end{array}$ & $\begin{array}{c}226.8 \pm 59.0 \\
(76-311)\end{array}$ & $\begin{array}{c}17.9 \pm 16.3 \\
(1-58)\end{array}$ & $\begin{array}{c}22.9 \pm 16.1 \\
(1-63)\end{array}$ \\
\hline $\begin{array}{l}\text { Kennebunk } \\
\text { River }\end{array}$ & $\begin{array}{l}43^{\circ} 25.50^{\prime} \mathrm{N} \\
70^{\circ} 33.83^{\prime} \mathrm{W}\end{array}$ & 37 & 24 & 65 & $\begin{array}{c}4.5 \pm 3.1 \\
(1-10)\end{array}$ & $\begin{array}{c}219.9 \pm 56.9 \\
(137-307)\end{array}$ & $\begin{array}{c}263.8 \pm 72.6 \\
(140-430)\end{array}$ & $\begin{array}{c}19.6 \pm 15.9 \\
(3-49)\end{array}$ & $\begin{array}{c}40.0 \pm 33.5 \\
(3-156)\end{array}$ \\
\hline Nonesuch River & $\begin{array}{l}43^{\circ} 35.15^{\prime} \mathrm{N}, \\
70^{\circ} 27.89^{\prime} \mathrm{W}\end{array}$ & 19 & 11 & 58 & $\begin{array}{c}1.4 \pm 0.7 \\
(1-3)\end{array}$ & $\begin{array}{c}206.4 \pm 45.9 \\
(161-285)\end{array}$ & $\begin{array}{c}233.5 \pm 62.5 \\
(125-311)\end{array}$ & $\begin{array}{c}13.8 \pm 11.5 \\
(6-39)\end{array}$ & $\begin{array}{c}26.0 \pm 19.0 \\
(2-59)\end{array}$ \\
\hline Kennebec River & $\begin{array}{l}43^{\circ} 54.82^{\prime} \mathrm{N}, \\
69^{\circ} 48.26^{\prime} \mathrm{W}\end{array}$ & 25 & 15 & 60 & $\begin{array}{c}3.7 \pm 3.2 \\
(1-11)\end{array}$ & $\begin{array}{c}291.4 \pm 44.7 \\
(242-364)\end{array}$ & $\begin{array}{c}304.6 \pm 59.7 \\
(246-445)\end{array}$ & $\begin{array}{c}40.3 \pm 16.2 \\
(22-72)\end{array}$ & $\begin{array}{c}51.7 \pm 32.2 \\
(25-136)\end{array}$ \\
\hline St. George River & $\begin{array}{l}44^{\circ} 07.41^{\prime} \mathrm{N}, \\
69^{\circ} 14.80^{\prime} \mathrm{W}\end{array}$ & 49 & 17 & 35 & $\begin{array}{c}1.9 \pm 1.1 \\
(1-4)\end{array}$ & $\begin{array}{c}180.9 \pm 76.3 \\
(67-343)\end{array}$ & $\begin{array}{c}231.2 \pm 88.7 \\
(71-361)\end{array}$ & $\begin{array}{c}15.1 \pm 17.2 \\
(1-69)\end{array}$ & $\begin{array}{c}30.9 \pm 25.4 \\
(1-76)\end{array}$ \\
\hline Medomak River & $\begin{array}{l}44^{\circ} 08.70^{\prime} \mathrm{N} \\
69^{\circ} 24.95^{\prime} \mathrm{W}\end{array}$ & 33 & 0 & 0 & 0 & $\begin{array}{c}317.5 \pm 106.8 \\
(126-531)\end{array}$ & $\begin{array}{l}- \\
-\end{array}$ & $\begin{array}{c}71.4 \pm 66.5 \\
(3-246)\end{array}$ & $\begin{array}{l}- \\
-\end{array}$ \\
\hline Ducktrap River & $\begin{array}{l}44^{\circ} 19.76^{\prime} \mathrm{N} \\
69^{\circ} 03.65^{\prime} \mathrm{W}\end{array}$ & 14 & 0 & 0 & 0 & $\begin{array}{c}303.2 \pm 124.3 \\
(101-510)\end{array}$ & - & $\begin{array}{c}67.8 \pm 77.2 \\
(1-254)\end{array}$ & $\begin{array}{l}- \\
-\end{array}$ \\
\hline Sheepscot River & $\begin{array}{l}44^{\circ} 21.52^{\prime} \mathrm{N}, \\
69^{\circ} 32.71^{\prime} \mathrm{W}\end{array}$ & 16 & 5 & 31 & $\begin{array}{c}1.6 \pm 0.9 \\
(1-3)\end{array}$ & $\begin{array}{c}321.9 \pm 99.5 \\
(176-500)\end{array}$ & $\begin{array}{c}355 \pm 144.3 \\
(150-507)\end{array}$ & $\begin{array}{c}65.5 \pm 61.5 \\
(6-221)\end{array}$ & $\begin{array}{c}105.3 \pm 99.7 \\
(5-228)\end{array}$ \\
\hline Union River & $\begin{array}{l}44^{\circ} 32.00^{\prime} \mathrm{N}, \\
68^{\circ} 24.60^{\prime} \mathrm{W}\end{array}$ & 19 & 0 & 0 & 0 & $\begin{array}{c}289.2 \pm 102.4 \\
(97-445)\end{array}$ & $\begin{array}{l}- \\
-\end{array}$ & $\begin{array}{c}56.2 \pm 48.5 \\
(2-175)\end{array}$ & $\begin{array}{l}- \\
-\end{array}$ \\
\hline
\end{tabular}


Table 1. (continued)

\begin{tabular}{|c|c|c|c|c|c|c|c|c|c|}
\hline \multirow[t]{2}{*}{ Location } & \multirow{2}{*}{$\begin{array}{l}\text { Lat., } \\
\text { Long. }\end{array}$} & \multicolumn{2}{|c|}{ No. of eels (n) } & \multirow{2}{*}{$\begin{array}{c}\text { Prevalence } \\
(\%)\end{array}$} & \multirow[t]{2}{*}{ Intensity } & \multicolumn{2}{|c|}{ Eel length (mm) } & \multicolumn{2}{|c|}{ Eel weight (g) } \\
\hline & & Collected & Infected & & & Non-infected & Infected & Non-infected & Infected \\
\hline Tunk Stream & $\begin{array}{l}44^{\circ} 36.49^{\prime} \mathrm{N} \\
67^{\circ} 59.75^{\prime} \mathrm{W}\end{array}$ & 10 & 0 & 0 & 0 & $\begin{array}{c}303.5 \pm 130.8 \\
(111-595)\end{array}$ & $\begin{array}{l}- \\
-\end{array}$ & $\begin{array}{c}84.1 \pm 124.8 \\
(2-427)\end{array}$ & $\begin{array}{l}- \\
-\end{array}$ \\
\hline Pleasant River & $\begin{array}{l}44^{\circ} 41.73^{\prime} \mathrm{N} \\
67^{\circ} 44.52^{\prime} \mathrm{W}\end{array}$ & 27 & 0 & 0 & 0 & $\begin{array}{c}229 \pm 78.5 \\
(95-345)\end{array}$ & $\begin{array}{l}- \\
-\end{array}$ & $\begin{array}{c}26.7 \pm 24.2 \\
(1-82)\end{array}$ & $\begin{array}{l}- \\
-\end{array}$ \\
\hline Penobscot River & $\begin{array}{l}44^{\circ} 45.31^{\prime} \mathrm{N} \\
68^{\circ} 46.17^{\prime} \mathrm{W}\end{array}$ & 19 & 5 & 26 & $\begin{array}{l}1.4 \pm 0.9 \\
(1-3)\end{array}$ & $\begin{array}{c}219.1 \pm 167.5 \\
(86-545)\end{array}$ & $\begin{array}{c}386.2 \pm 173.2 \\
(102-561)\end{array}$ & $\begin{array}{c}48.2 \pm 77.7 \\
(1-255)\end{array}$ & $\begin{array}{c}142.2 \pm 124.7 \\
(1-335)\end{array}$ \\
\hline $\begin{array}{l}\text { East Machias } \\
\text { River }\end{array}$ & $\begin{array}{l}45^{\circ} 00.05^{\prime} \mathrm{N} \\
67^{\circ} 37.09^{\prime} \mathrm{W}\end{array}$ & 15 & 0 & 0 & 0 & $\begin{array}{c}364.2 \pm 74.6 \\
(247-551)\end{array}$ & $\begin{array}{l}- \\
-\end{array}$ & $\begin{array}{c}95.8 \pm 74.5 \\
(25-320)\end{array}$ & $\begin{array}{l}- \\
-\end{array}$ \\
\hline Nova Scotia & & & & & & & & & \\
\hline West River & $\begin{array}{l}43^{\circ} 44.71^{\prime} \mathrm{N} \\
65^{\circ} 09.40^{\prime} \mathrm{W}\end{array}$ & 24 & 0 & 0 & 0 & $\begin{array}{c}261.7 \pm 32.2 \\
(175-307)\end{array}$ & $\begin{array}{l}- \\
-\end{array}$ & $\begin{array}{c}30.4 \pm 10.9 \\
(10-45)\end{array}$ & - \\
\hline Chebogue River & $\begin{array}{l}43^{\circ} 50.48^{\prime} \mathrm{N} \\
66^{\circ} 03.49^{\prime} \mathrm{W}\end{array}$ & 19 & 0 & 0 & 0 & $\begin{array}{c}251.0 \pm 73.8 \\
(110-335)\end{array}$ & - & $\begin{array}{c}36.6 \pm 24.80 \\
(5-80)\end{array}$ & $\begin{array}{l}- \\
-\end{array}$ \\
\hline Beaver River $^{a}$ & $\begin{array}{l}43^{\circ} 59.89^{\prime} \mathrm{N} \\
66^{\circ} 08.73^{\prime} \mathrm{W}\end{array}$ & 78 & 0 & 0 & 0 & $\begin{array}{c}273.1 \pm 56.3 \\
(160-480)\end{array}$ & $\begin{array}{l}- \\
-\end{array}$ & $\begin{array}{c}39.5 \pm 29.9 \\
(5-215)\end{array}$ & $\begin{array}{l}- \\
-\end{array}$ \\
\hline Salmon River ${ }^{\mathrm{a}}$ & $\begin{array}{l}44^{\circ} 03.29^{\prime} \mathrm{N} \\
66^{\circ} 09.17^{\prime} \mathrm{W}\end{array}$ & 81 & 0 & 0 & 0 & $\begin{array}{c}284.0 \pm 69.0 \\
(110-465)\end{array}$ & - & $\begin{array}{l}45.3 \pm 30.4 \\
\quad(5-140)\end{array}$ & - \\
\hline Metaghan River ${ }^{a}$ & $\begin{array}{l}44^{\circ} 12.71^{\prime} \mathrm{N} \\
66^{\circ} 07.88^{\prime} \mathrm{W}\end{array}$ & 77 & 0 & 0 & 0 & $\begin{array}{c}261.2 \pm 39.9 \\
(110-327)\end{array}$ & - & $\begin{array}{c}33.2 \pm 14.5 \\
\quad(5-70)\end{array}$ & $\begin{array}{l}- \\
-\end{array}$ \\
\hline $\begin{array}{l}\text { Grosses Coques } \\
\text { River }^{\mathrm{a}}\end{array}$ & $\begin{array}{l}44^{\circ} 22.47^{\prime} \mathrm{N} \\
66^{\circ} 04.47^{\prime} \mathrm{W}\end{array}$ & 47 & 0 & 0 & 0 & $\begin{array}{c}247.1 \pm 72.7 \\
(80-360)\end{array}$ & - & $\begin{array}{c}31.6 \pm 24.3 \\
(5-105)\end{array}$ & $\begin{array}{l}- \\
-\end{array}$ \\
\hline Sissiboo River & $\begin{array}{l}44^{\circ} 24.56^{\prime} \mathrm{N} \\
65^{\circ} 59.76^{\prime} \mathrm{W}\end{array}$ & 33 & 0 & 0 & 0 & $\begin{array}{c}223.9 \pm 54.9 \\
(137-347)\end{array}$ & & $\begin{array}{l}24.6 \pm 22.3 \\
(5-100)\end{array}$ & \\
\hline Ontario & & & & & & & & & \\
\hline $\begin{array}{l}\text { Moses-Saunders } \\
\text { Dam }^{\mathrm{b}}\end{array}$ & $\begin{array}{l}45^{\circ} 00.35^{\prime} \mathrm{N} \\
74^{\circ} 47.78^{\prime} \mathrm{W}\end{array}$ & 60 & 0 & 0 & 0 & $\begin{array}{c}367.8 \pm 70.6 \\
(250-610)\end{array}$ & - & $\begin{array}{c}66.9 .8 \pm 53.5 \\
(10-315)\end{array}$ & $\begin{array}{l}- \\
-\end{array}$ \\
\hline Quebec & & & & & & & & & \\
\hline $\begin{array}{l}\text { Beauharnois } \\
\text { Dam }^{b}\end{array}$ & $\begin{array}{l}45^{\circ} 18.89^{\prime} \mathrm{N} \\
73^{\circ} 54.82^{\prime} \mathrm{W}\end{array}$ & 25 & 0 & 0 & 0 & $\begin{array}{c}336.2 \pm 66.4 \\
(245-450)\end{array}$ & $\begin{array}{l}- \\
-\end{array}$ & $\begin{array}{c}55.4 .0 \pm 33.0 \\
(20-120)\end{array}$ & $\begin{array}{l}- \\
-\end{array}$ \\
\hline Verte River ${ }^{\mathrm{b}}$ & $\begin{array}{l}48^{\circ} 00.44^{\prime} \mathrm{N} \\
69^{\circ} 20.75^{\prime} \mathrm{W}\end{array}$ & 9 & 0 & 0 & 0 & $\begin{array}{c}218.3 \pm 131.9 \\
(75-405)\end{array}$ & - & $\begin{array}{c}38.2 \pm 45.0 \\
(4-110)\end{array}$ & $\begin{array}{l}- \\
-\end{array}$ \\
\hline Trois Pistoles $^{\mathrm{b}}$ & $\begin{array}{l}48^{\circ} 05.73^{\prime} \mathrm{N} \\
69^{\circ} 12.79^{\prime} \mathrm{W}\end{array}$ & 13 & 0 & 0 & 0 & $\begin{array}{c}367.7 \pm 163.5 \\
(110-675)\end{array}$ & $\begin{array}{l}- \\
-\end{array}$ & $\begin{array}{c}155.4 \pm 201.4 \\
(5-695)\end{array}$ & $\begin{array}{l}- \\
-\end{array}$ \\
\hline $\begin{array}{l}\text { Riviere } \\
\text { Sud-Ouest }^{\mathrm{b}}\end{array}$ & $\begin{array}{l}48^{\circ} 21.12^{\prime} \mathrm{N} \\
68^{\circ} 45.68^{\prime} \mathrm{W}\end{array}$ & 22 & 0 & 0 & 0 & $\begin{array}{c}311.4 \pm 92.4 \\
(185-650)\end{array}$ & $\begin{array}{l}- \\
-\end{array}$ & $\begin{array}{c}68.2 \pm 104.9 \\
(10-525)\end{array}$ & $\begin{array}{l}- \\
-\end{array}$ \\
\hline
\end{tabular}

Although the survey of the St. Lawrence River system was not exhaustive, it was comprehensive in its coverage and should have detected the parasite if present. The 3 tributaries within Quebec are representative of the lower St. Lawrence region, while the other 2 sites (Beauharnois and Moses-Saunders eel ladders) consisted of samples of upstream migrating eels Anguilla rostrata that were at least 6 yr old (K. Oliveira unpubl. data on age). The absence of the parasite in some sampled locations is probably not due to differences in eel size distribution. All eel collections in the present study included eels of multiple size classes, which should have allowed the detection of the parasite, if it had been present at each site.

All of the sites in the present study were sampled in the summer months, and Maine, in particular, was sampled within a $2 \mathrm{wk}$ period. During this time the parasite is presumably most active and infective. A study of infection in the Elbe River in Germany did not depict any clear seasonal fluctuations in the parasite prevalence (Möller et al. 1991). Although parasite seasonality does not appear to have masked the presence of adult nematodes at the northernmost sites, it cannot be ruled out. Further study using a temporally staggered sampling design would be required to determine whether parasite prevalence varies seasonally.

The distribution of Anguillicola crassus in yellow eels collected from sites throughout New England depicted no significant correlation between latitude and the prevalence of infection, as has been noted in a previous study of American eels in North Carolina (Moser et al. 2001). The distribution and range may be the result of the parasite having $>1$ transport mechanism. The current range of $A$. crassus may be related to the movement and distribution of intermediate and paratenic hosts, which are believed to be the main 
Table 2. Anguillicola crassus, Anguilla rostrata. Prevalence and intensity of A. crassus infection in eels A. rostrata collected within the commercial fishery from New Brunswick and northern Nova Scotia. The Prince Edward Island and Newfoundland eels were only available through swim bladder samples of eels collected from these provinces. All eels were $\geq 300 \mathrm{~mm}$ total length

\begin{tabular}{|c|c|c|c|c|}
\hline $\begin{array}{l}\text { River } \\
\text { (Location) }\end{array}$ & $\begin{array}{l}\text { Numbe } \\
\text { Collected }\end{array}$ & $\begin{array}{l}\text { of eels } \\
\text { Infected }\end{array}$ & $\begin{array}{c}\text { Prevalence } \\
(\%)\end{array}$ & Intensity \\
\hline \multicolumn{5}{|l|}{ New Brunswick } \\
\hline St. Johns River & 28 & 1 & 3.6 & 1.0 \\
\hline Silver Lake (Amherst) & 32 & 1 & 3.1 & 1.0 \\
\hline \multicolumn{5}{|l|}{ Nova Scotia } \\
\hline Lochabere Lake (Antigonish) & 27 & 4 & 14.8 & 2.5 \\
\hline Margaree Harbor (Cape Breton) & 26 & 8 & 30.1 & 6.1 \\
\hline Bras d'Or Lake (Cape Breton) & 28 & 4 & 14.3 & 4.0 \\
\hline $\begin{array}{l}\text { Prince Edward Island } \\
\text { Malpeque Bay } \\
\text { (Northwest of Charlottetown) }\end{array}$ & 146 & 0 & 0.0 & 0.0 \\
\hline $\begin{array}{l}\text { Newfoundland } \\
\text { Bonavista Bay } \\
\text { (Southwest of Gander) }\end{array}$ & 133 & 0 & 0.0 & 0.0 \\
\hline
\end{tabular}

mechanisms behind the parasite's rapid dispersal in European eels considering the extensive range of both types of transitional hosts (Moravec 1992, Moravec \& Skoríková 1998). Deep snout pipefish and black goby have been naturally infected with nematodes in the Baltic Sea, confirming freshwater, estuarine, and marine infections (Reimer et al. 1994). This supports the hypothesis that the parasite is not restricted to freshwater and can potentially be transferred to new locations through all salinity regimes and through many paratenic hosts (reviewed by Taraschewski 2006). The vast array of potential hosts and the eel's generalist feeding behavior (Helfman et al. 1987, Barak \& Mason 1992) ensure that the parasite's distribution will not be limited by host availability. Yellow American eels exhibit homing, home site fidelity, and limited home ranges (Parker 1995, Oliveira 1997, Morrison \& Secor 2003), and thus their movements are unlikely to contribute to the parasite's spread between river systems. It is also conceivable that the current distribution is related to anthropogenic mechanisms. Although natural eel movement may not enhance parasite spread between rivers, eels sold for live bait may do so. Live eels are transported between rivers as bait, primarily to supply recreational fisheries, potentially spreading the parasite to an entirely different river system from that in which the eel originated. In addition, 6 of the 8 areas with the highest prevalence and greatest intensity in the present study were in the vicinity of major shipping ports (Boston, Massachussetts, New Bedford, Massachussetts, and Portland, Maine). In a study of the exotic American eel gill parasites Pseudodactylogyrus bini and $P$. anguillae, it was hypothesized that they were introduced along with $A$. crassus through either commercial imports of foreign eels or by transmission through ballast water (Hayward et al. 2001). Machut \& Limburg (2008) found increased infection rates in tributaries of the Hudson River that exhibited higher urbanization, indicating that anthropogenically induced eel susceptibility to the parasite may also be a factor involved in parasite distribution. The presence of the parasite in the St. Johns River can be explained by the natural advancing of the parasite along the coast from New England rivers. This collection was conducted 2 yr after the parasite had been found within the Penobscot River drainage approximately $180 \mathrm{~km}$ away. However, the presence of the parasite in northern New Brunswick and Nova Scotia, while being absent in the southern portion of Nova Scotia, is problematic and indicative of a secondary mode of transport.

The distribution of Anguillicola crassus found in the present study does not appear to be limited by temperature, as has been suggested in other studies (Knopf et al. 1998). Eels from the Penobscot River drainage are infected with A. crassus, and this system has similar annual water temperatures to the East Machias River, Maine, the northernmost site sampled within the USA, and to the southern Nova Scotia rivers. Knopf et al. (1998) proposed that American eels of the St. Lawrence River would not be affected by a northward expansion because low winter water temperatures would hinder the parasite's survival. They also state that $\mathrm{L}_{3}$ stage larvae can withstand $4^{\circ} \mathrm{C}$ for periods up to $4 \mathrm{mo}$, with low temperature presumably causing delayed development and high mortality of the adult parasite phase within the eel. However, the parasite is well established in Penobscot River eels, which are subjected to mean wa-

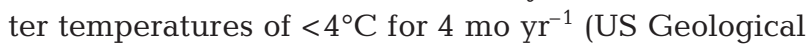
Survey 2001). The presence of the parasite in northern Nova Scotia and New Brunswick does not support this temperature-limiting hypothesis either, and it is probable that the parasite will eventually be found further north, possibly entering the St. Lawrence River system, before temperature becomes an actual limiting factor.

As a panmictic species (Avise et al. 1986, Wirth \& Bernatchez 2003), the spread of the parasite into more northern locations will only add to the eel's current recruitment problems. Further study of Anguillicola crassus is thus necessary to better understand the mechanisms determining its distribution and rate of dispersal, in order to assess the future effect of this parasite on the American eel. 
Acknowledgements. This research was funded in part by a grant to K. Oliveira from the United States Fish and Wildlife Service's Fish Enhancement, Mitigation, and Research Fund under Project 2005-0129-003. We thank W. Krueger for reading an earlier draft of this manuscript, P. Aieta, D. Aieta, F. Boulougouras, J. Dayton and S. Oliveira for their assistance in much of the field collections, and M. Feingenbaum and his staff from South Shore Trading for assisting us in sampling eels from his facility.

\section{LITERATURE CITED}

Avise JC, Helfman GS, Saunders NC, Hales LS (1986) Mitochondrial DNA differentiation in North Atlantic eels: population genetic consequences of an unusual life history pattern. Proc Natl Acad Sci USA 83:4350-4354

Barak NAE, Mason CF (1992) Population density, growth and diet of eels, Anguilla anguilla L., in two rivers in eastern England. Aquacult Fish Manag 23:59-70

Barse AM, Secor D (1999) An exotic nematode parasite of the American eel. Fisheries 24:6-10

- Bush AO, Lafferty KD, Lotz JM, Shostaki AW (1997) Parasitology meets ecology on its own terms: Margolis et al. revisited. J Parasitol 83:575-583

Castonguay M, Hodson PV, Couillard CM (1994) Why is recruitment of the American eel, Anguilla rostrata, declining in the St. Lawrence River and Gulf? Can J Fish Aquat Sci 51:479-488

> De Charleroy D, Grisez L, Thomas K, Belpaire C, Ollevier F (1990) The life cycle of Anguillicola crassus. Dis Aquat Org 8:77-84

Friedland KD, Miller MJ, Knights B (2007) Oceanic changes in the Sargasso Sea and declines in recruitment of the European eel. ICES J Mar Sci 64:519-530

Fries LT, Williams DJ, Johnson KS (1996) Occurrence of Anguillicola crassus, an exotic parasitic swim bladder nematode of eels, in the southeastern United States. Trans Am Fish Soc 125:794-797

Gollock MJ, Kennedy CR, Quabius E, Brown JA (2004) The effect of parasitism of European eels with the nematode Anguillicola crassus on the impact of netting and aerial exposure. Aquaculture 233:45-54

Haro A, Richkus W, Whalen K, Hoar A and others (2000) Population decline of the American eel: implications for research and management. Fisheries 25:7-16

Hayward CJ, Iwashita M, Crane JS, Ogawa K (2001) First report of the invasive eel pest Pseudodactylogyrus bini in North America and in wild American eels. Dis Aquat Org 44:53-60

Helfman GS, Facey DE, Hales LS Jr, Bozeman EL (1987) Reproductive ecology of the American eel. Am Fish Soc Symp 1:42-56

Johnson SK, Fries LT, Williams J, Huffman DG (1995) Presence of the parasitic swim bladder nematode, Anguillicola crassus, in Texas aquaculture. World Aquacult 26:35-36

Kirk RS (2003) The impact of Anguillicola crassus on European eels. Fish Manag Ecol 10:385-394

Knopf K, Würtz J, Sures B, Taraschewski H (1998) Impact of low water temperature on the development of Anguillicola crassus in the final host Anguilla anguilla. Dis Aquat Org 33:143-149

Kuwahara A, Niimi A, Itagaki H (1974) Studies on a nematode parasitic in the air bladder of the Japanese eel. J Parasitol 23:275-279

Machut LS, Limburg KE (2008) Anguillicola crassus infection in Anguilla rostrata from small tributaries of the Hudson River watershed, New York, USA. Dis Aquat Org 79: 37-45
Mellergaard S, Dalsgaard I (1989) Handbook of eel diseases. Damn Fisk og Havunders Rapport 293, p 1-47

Möller H, Holst S, Lüchtenberg H, Peterson F (1991) Infection of eel Anguilla anguilla from the river Elbe estuary with two nematodes, Anguillicola crassus and Pseudoterranova decipiens. Dis Aquat Org 11:193-199

- Molnár K (1994) Formation of parasitic nodules in the swimbladder and intestinal walls of the eel Anguilla anguilla due to infections with larval stages of Anquillicola crassus. Dis Aquat Org 20:163-170

Moravec F (1992) Spreading of the nematode Anguillicola crassus (Dracuncoloidea) among eel populations in Europe. Folia Parasitol (Praha) 39:247-248

> Moravec F (2004) Some aspects of the taxonomy and biology of drancunculoid nematodes parasitic in fishes: a review. Folia Parasitol (Praha) 51:1-13

Moravec F, Skoríková B (1998) Amphibians and larvae of aquatic insects as new paratenic hosts of Anguillicola crassus (Nematoda: Dracunculoidea), a swimbladder parasite of eels. Dis Aquat Org 34:217-222

> Moravec F, Taraschewski H (1988) Revision of the genus Anguillicola Yamaguti, 1935 (Nematoda: Anguillicolidae) of the swimbladder of eels, including a description of two new species, A. novaezelandiae sp. n. and A. papernai sp. n. Folia Parasitol (Praha) 35:125-146

Morrison W, Secor D (2003) Demographic attributes of yellow-phase American eels (Anguilla rostrata) in the Hudson River estuary. Can J Fish Aquat Sci 60:1487-1501

> Moser ML, Patrick WS, Crutchfield JU Jr (2001) Infection of American eels, Anguilla rostrata, by an introduced nematode parasite, Anguillicola crassus, in North Carolina. Copeia 2001:848-853

Oliveira K (1997) Movements and growth rates of yellowphased American eels in the Annaquatucket River, Rhode Island. Trans Am Fish Soc 126:638-646

Ooi HK, Wang WS, Chang HY, Wu CH, Lin CC, Hsieh MT (1996) An epizootic of Anguillicolosis in cultured American eels in Taiwan. J Aquat Anim Health 8:163-166

Parker SJ (1995) Homing ability and home range of yellowphased American eels in a tidally dominated estuary. J Mar Biol Assoc UK 75:127-140

Pietrock M, Meinelt T (2002) Dynamics of Anguillicola crassus larval infections in a paratenic host, the ruffe (Gymnocephalus cernuus) from the Oder River on the border of Germany and Poland. J Helminthol 76:235-240

- Reimer LW, Hildebrand A, Scharberth D, Walter U (1994) Anguillicola crassus in the Baltic Sea: field data supporting transmission in brackish waters. Dis Aquat Org 18: 77-79

Richkus W, Whalen K (1999) American eel (Anguilla rostrata) scoping study: a literature and data review of life history, stock status, population dynamics, and hydroelectric impacts. TR-111873, Electric Power Research Institute, Palo Alto, CA

Sokolowski MS, Dove ADM (2006) Histopathological examination of wild American eels infected with Anguillicola crassus. J Aquat Anim Health 18:257-262

Taraschewski H (2006) Hosts and parasites as aliens. J Helminthol 80:99-128

Tesch FW (2003) The eel, 5th edn. Blackwell Science, Oxford

US Geological Survey (2001) National water information system (NWISWeb) data. Available at: http://waterdata.usgs. gov/me/nwis/monthly (accessed on 30 March 2008)

Wirth T, Bernatchez L (2003) Decline of North Atlantic eels: a fatal synergy? Proc R Soc Lond B 270:681-688

> Würtz J, Taraschewski H (2000) Histopathological changes in the swimbladder wall of the European eel Anguilla anguilla due to infections with Anguillicola crassus. Dis Aquat Org 39:121-134 